\title{
Impact of nanoclay on the properties of wood polymer nanocomposites
}

\begin{abstract}
In this chapter, the influences of nanoclay on the attributes of tropical wood polymer nanocomposites (WPNCs) were discussed. The impregnation of wood samples with nanoclay and in situ polymerization were employed by vacuum-pressure method. The comparisons of improvement in different properties have been showed between the raw wood, wood plastic composites (WPC), and WPNC. The FTIR result confirmed the interaction and incorporation of nanoclay inside wood. The exfoliation of nanoclay has been identified by Transmission electron microscopy result. The formation of wood polymer nanocomposites was proven through X-ray diffraction (XRD) pattern and Scanning electron microscopy (SEM) images, respectively. Significant improvement in mechanical properties was found through nanoclay treatment. The Dynamic mechanical thermal analysis (DMTA) result also indicated the improvement of thermo-mechanical properties after nanoclay treatment. The storage modulus $\left(E^{\prime}\right)$ of the WPNC specimen also unveiled notable enhancement in both rubbery plateau and glassy region in alliance to their parallel wood polymer composites and raw wood samples. The wood's Dynamic Young's modulus (Ed) also indicated significant increment for PFnanoclay impregnated WPNC samples. Whereas, the treatment by PF-nanoclay system seem to lower the damping (loss Tan $\delta$ ) peaks of woods. Furthermore, the WPNC samples shown significant improved compared to WPC and raw ones against white-rot white-rot (polyporous versicolor) and brown-rot (postia placenta) exposed.
\end{abstract}

Keyword: Nanoclay; Wood polymer nanocomposites; Scanning electron microscopy; Dynamic mechanical thermal analysis; Fourier transform infrared spectroscopy 